\title{
Eficiência e deposição de herbicidas na cebola em função do adjuvante e da taxa de aplicação
}

\author{
Efficiency and deposition of herbicides in onion in function of adjuvant \\ and carrier volume
}

\begin{abstract}
Antonio Mendes de Oliveira Neto ${ }^{1 *}$, Gustavo Cunha ${ }^{2}$, Dieison Olescowicz², Tainá Gutz², Marlon Goede², Oscar Emílio Ludtke Harthmann², Naiara Guerra ${ }^{3}$
\end{abstract}

Resumo - Objetivou-se, com este trabalho, avaliar o efeito da taxa de aplicação e do uso de adjuvante sobre o controle de plantas daninhas e deposição dos herbicidas ioxynil + clethodim em plantas de cebola. O experimento consistiu em seis tratamentos com cinco repetições, arranjados em esquema fatorial 2 x 3, ou seja, a combinação de dois níveis de adjuvante (com e sem) com três taxas de aplicação $\left(100,150\right.$ e $\left.200 \mathrm{~L} \mathrm{ha}^{-1}\right)$. Os tratamentos foram aplicados em pós-emergência, após o enraizamento da cebola e quando as plantas daninhas se apresentavam no estádio de quatro a seis folhas. Os tratamentos não promoveram fitotoxicidade na cultura da cebola. Houve tendência de melhoria no controle de Cyclospermum leptophyllum e Polygonum persicaria com o uso do adjuvante, contudo não houve significância. A deposição de calda não foi afetada pela taxa de aplicação e pelo uso de adjuvante. Conclui-se que o uso de adjuvante e a redução da taxa de aplicação não comprometeram a eficiência e a deposição dos herbicidas ioxynil + clethodim.

Palavras-chave: Cyclospermum leptophyllum, óleo mineral, Polygonum persicaria

\begin{abstract}
The objective, of this work, was evaluated the effect of carrier volume and use of activator adjuvant on the weed control and deposition of herbicide treatment with ioxynil + clethodim in onion plants. The experiment consisted by six treatments and five replications, organized in factorial scheme $2 \times 3$, that is a combination of two levels of adjuvant (with an without) with three application rates $\left(100,150\right.$ e $\left.200 \mathrm{~L} \mathrm{ha}^{-1}\right)$. The treatments were applicate in post-emergence, after the onion rooted in and the weeds were four to six leaves stage. The treatments not promote intoxication in onion crop. There are the tendency to improve Cyclospermum leptophyllum and Polygonum persicaria control with adjuvant use, however without significate effect. The control level and deposition were not affect for application rate evaluated. Concluded that reduced application rate no compromised the efficiency and deposition of ioxynil + clethodim herbicides.
\end{abstract}

Keywords: Cyclospermum leptophyllum, mineral oil, Polygonum persicaria

Recebido: Abril 03, 2018. Aceito: Fevereiro 02, 2019

${ }^{1}$ Departamento de Agronomia, Centro de Ciências Agroveterinárias, Universidade do Estado de Santa Catarina - UDESC, Avenida Luiz de Camões, 2090, Conta Dinheiro, CEP 88520-000, Lages, SC, Brasil.

E-mail: antonio.neto@udesc.br

${ }^{2}$ Instituto Federal Catarinense - IFC, Rio do Sul, SC, Brasil. E-mail: agronomia.cunha@gmail.com; dieisonoles@gmail.com; tainagutz.tg@gmail.com; marlongoed@hotmail.com; oscar.harthmann@ifc.edu.br

${ }^{3}$ Coordenadoria Especial de Ciências Biológicas e Agronômicas, Universidade Federal de Santa Catarina - UFSC, Curitibanos, SC, Brasil. E-mail: naiara.guerra@ufsc.br 


\section{Introdução}

Muitos problemas estão relacionados à diminuição da qualidade de bulbos e produtividade na cultura da cebola (Allium cepa), dentre os quais a interferência causada pelas plantas daninhas figura entre os mais relevantes, (Epagri, 2013). Algumas características da cebola, como o crescimento inicial lento, disposição foliar ereta, folhas com forma cilíndrica, porte baixo e consequentemente baixa capacidade de sombreamento do solo, fazem com que sua competitividade com as plantas daninhas seja baixa em qualquer fase do desenvolvimento da cultura (Ferreira et al., 1999; Ferreira et al., 2000; Soares et al., 2003).

$\mathrm{Na}$ tentativa de evitar a interferência imposta pelas plantas daninhas, os cebolicultores optam predominantemente pelo controle químico como o principal método de controle de plantas daninhas. Esta escolha é fortemente influenciada pela elevada eficiência, praticidade, menor custo, baixo requerimento de mão de obra, menor dependência do clima, flexibilidade de aplicação, menor dano ao sistema radicular e possibilidade de uso em diferentes arranjos de plantas (Durigan et al., 2005). Contudo, apesar da ampla utilização, o controle químico de plantas daninhas em cebola ainda necessita de maior aprimoramento, principalmente no que diz respeito à tecnologia de aplicação adotada.

Neste contexto, os cebolicultores da região do Alto Vale do Itajaí, SC comumente utilizam taxa de aplicação entre 300 e $400 \mathrm{~L} \mathrm{ha}^{-1} \mathrm{em}$ todas as aplicações, independentemente do produto fitossanitário utilizado. Pesquisas que envolvem essa temática na cebola são escassas. Um trabalho pioneiro realizado por Gonçalves e Palladini (2000) avaliou o efeito do uso de modelos de pontas de pulverização e taxa de aplicação sobre o controle de tripes (Thrips tabaci) na cultura da cebola, esses autores concluíram que as pontas cone (CONEJET TXVS 8, CONEJET TXVK 18, CONEJET TXVK 26 e D6 DIFUSOR V5) e leque (XR 110015 VS, XR 11002 VS, XR 11003 VS,
XR 11004 VS, XR 11005 VS, TJ60 11002 VS e TJ60 11004 VS), aplicando taxas variando de 236 a $788 \mathrm{~L} \mathrm{ha}^{-1}$, apresentaram a mesma eficiência no controle de tripes em cebola.

Os adjuvantes podem melhorar a eficiência dos herbicidas por modificarem determinadas propriedades físico-químicas da calda. Neste sentido, o grupo dos surfactantes são eficientes em reduzir a tensão superficial da calda, favorecendo a cobertura foliar, sendo o efeito sobre a deriva dependente do surfactante utilizado, podendo reduzir, não ter nenhum efeito ou aumentá-la. Já os óleos minerais são eficientes em reduzir a tensão superficial da calda e minimizar o percentual de deriva da aplicação. Os óleos vegetais por sua vez, reduzem a tensão superficial da calda, aumentam o diâmetro mediano volumétrico (DMV) e reduzirem as perdas de gotas por deriva (Oliveira, 2011). As alterações promovidas pelos adjuvantes podem ser benéficas para a eficiência da aplicação, já que favorecem a maior cobertura do alvo e, ou minimizam o transporte de gotas para fora do alvo biológico.

Atualmente, estão disponíveis pontas de pulverização, que podem ser utilizadas com baixa pressão (100 a $300 \mathrm{kPa}$ ), possibilitando a redução da taxa de aplicação, além de reduzir o potencial de deriva, reduzindo a proporção de gotas com diâmetro $<100 \mu \mathrm{m}$ (Boller e Machry, 2007). Por isso, na tentativa de otimizar o uso de água e o rendimento operacional, formulou-se a hipótese inicial de que seria possível reduzir a taxa de aplicação de herbicidas na cebola, utilizando pontas de pulverização que reduzem o potencial de deriva e óleo mineral para favorecer a deposição da calda, sem que haja prejuízos ao controle de plantas daninhas.

Desta forma a pesquisa foi realizada com o objetivo de avaliar o efeito da taxa de aplicação e do uso de adjuvante sobre o controle de plantas daninhas e deposição dos herbicidas ioxynil + clethodim na cultura da cebola. 


\section{Material e métodos}

O experimento foi conduzido em área comercial de produção de cebola, no município de Aurora, SC. A precipitação observada durante o período do experimento está apresentada na Figura 1. O delineamento experimental adotado foi o de blocos ao acaso com seis tratamentos e cinco repetições, totalizando 30 unidades experimentais. Os tratamentos foram arranjados em esquema fatorial $2 \times 3$, avaliando-se o uso de adjuvante (sem ou com adjuvante na dose de $\left.0,5 \% \mathrm{~V} \mathrm{~V}^{-1}\right)$, combinado com três taxas de aplicação $\left(100,150\right.$ e $\left.200 \mathrm{Lha}^{-1}\right)$, sendo que o adjuvante utilizado foi o óleo mineral Nimbus (428 $\mathrm{g} \mathrm{L}^{-1}$ de óleo mineral, CE e Syngenta). Manteve-se uma testemunha adicional sem tratamento que foi utilizada como área de referência para avaliação de controle, contudo não fez parte do delineamento. Cada unidade experimental teve dimensão de $2 \times 3 \mathrm{~m}\left(6,0 \mathrm{~m}^{2}\right)$ e a área útil de cada parcela foi de $2,0 \mathrm{~m}^{2}$.

A cultura da cebola foi implantada por meio de mudas, com transplante ocorrendo no mês de julho. A cultivar utilizada foi a bola precoce (CEMADEN, 2018), em espaçamento entre linhas de $0,30 \mathrm{~m}$ e a densidade de 10 plantas por metro linear. As práticas culturais foram realizadas seguindo o manejo adotado pelo agricultor na área comercial, exceto o controle de plantas daninhas.

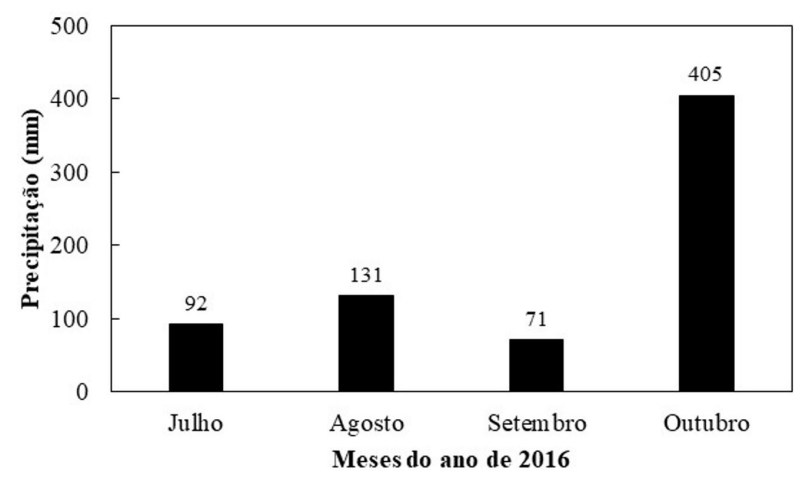

Figura 1. Precipitação (mm) observada durante o período de condução do experimento, dados foram obtido na Estação Pluviométrica de Ituporanga, SC.
O tratamento herbicida utilizado consistiu da associação de ioxynil a $250 \mathrm{~g} \mathrm{ha}^{-1}$ de i.a. + clethodim a $96 \mathrm{~g} \mathrm{ha}^{-1}$ de i.a. Os herbicidas comerciais utilizados foram Totril $\left(250 \mathrm{~g} \mathrm{~L}^{-1} \mathrm{de}\right.$ i.a., CE e Bayer) e Select $240 \mathrm{CE}\left(240 \mathrm{~g} \mathrm{~L}^{-1} \mathrm{de}\right.$ i.a., CE e Arysta), respectivamente. Em todos os tratamentos herbicidas adicionou-se o traçador azul brilhante-FD\&C 1 (Duas Rodas, Jaraguá do Sul, SC, Brasil) na concentração de $1.000 \mathrm{mg} \mathrm{L}^{-1}$ (Alves et al., 2014).

Os herbicidas foram aplicados em pós-emergência da cultura da cebola e das plantas daninhas, após o enraizamento da cebola e quando as plantas daninhas apresentavam quatro a seis folhas. Os tratamentos foram aplicados no dia 30/09/2016, com pulverizador costal pressurizado a $\mathrm{CO}_{2}$, com pressão constante de $207 \mathrm{kPa}$, barra com quatro pontas espaçadas a $0,5 \mathrm{~m}$ entre si e velocidade de deslocamento constante de $3,6 \mathrm{~km} \mathrm{~h}^{-1}$. As diferentes taxas de aplicação foram obtidas em função da diferença entre a vazão das pontas AD110.01, AD110.015 e AD 110.02. A pressão de trabalho, espaçamento entre bicos e a velocidade de deslocamento foram as mesmas em todas as aplicações. No momento da aplicação dos tratamentos a temperatura média do ar era de $18^{\circ} \mathrm{C}$, a umidade relativa do ar era de $56 \%$ e a velocidade do vento oscilou de 2,0 a $4,0 \mathrm{~km} \mathrm{~h}^{-1}$. A opção pelas pontas da série AD (Magno Jet) deve-se ao diâmetro das gotas geradas, com diâmetro mediano volumétrico (DMV) entre 250 e $300 \mu \mathrm{m}$ nas condições experimentais (informações disponibilizadas pelo fabricante).

Aos 15 dias após a aplicação dos tratamentos (DAA) realizou-se avaliação de eficiência de controle das plantas daninhas e de fitointoxicação para cultura da cebola. As avaliações de eficiência de controle das plantas daninhas foram realizadas com base em escala percentual, onde $0 \%$ representa nenhum controle e $100 \%$ a morte da planta (Kuva et al., 2016). Já as avaliações de fitointoxicação foram realizadas seguindo os critérios da EWRC (1964), em que se utiliza uma escala com intervalo de 1 a 9 , sendo que a nota 1,0 representa nenhum sintoma de fitointoxicação e 
a nota 9,0 representa a morte da planta. Os alvos biológicos avaliados foram Cyclospermum leptophyllum (cenourinha) e Polygonum persicaria (erva-de-bicho) em função da uniformidade de distribuição na área experimental. $\mathrm{Na}$ área havia infestação de Poa annua (pastinho-de-inverno) e Sisyrinchium fasciculatum (palminha), contudo a heterogeneidade de distribuição não permitiu a avaliação de eficiência.

Para avaliação de deposição repetiu-se o mesmo experimento em condições de casa de vegetação (em 17/08/2017) em vasos plásticos com capacidade volumétrica de $8 \mathrm{dm}^{3}$. Cada unidade experimental foi composta de quatro plantas de cebola com três folhas completamente expandidas e por uma lamínula posicionada no solo. Imediatamente após a aplicação realizou-se a coleta das plantas de cebola e das lamínulas, que foram conduzidas até o laboratório para recuperação do traçador, por meio de lavagem dupla com volume de $100 \mathrm{~mL}$ de água destilada. No dia seguinte (18/08/2017) realizou-se a quantificação do traçador por meio de um espectrofotômetro, utilizando o comprimento de onda de $630 \mathrm{~nm}$, faixa de detecção do azul, de acordo com a metodologia empregada por Palladini et al. (2005). As plantas de cebola foram secas em estufa de circulação forçada de ar a $65^{\circ} \mathrm{C}$, até a obtenção de massa constante. Os valores de deposição foram apresentados em $\mu \mathrm{L}$ de traçador por $\mathrm{g}^{-1}$ de massa seca e $\mu \mathrm{L}$ de traçador por $\mathrm{cm}^{-2}$ de lamínula.

Os dados foram submetidos a análise de variância pelo teste $\mathrm{F}$ e os fatores adjuvante (A) e volume de aplicação (VA) foram desdobrados, sendo que A foi comparado pelo teste F e VA comparado pelo teste de Tukey. Todas as análises foram realizadas a $5 \%$ de probabilidade $(\mathrm{p}<0,05)$.

\section{Resultados e discussão}

Não se visualizou sintomas visuais de fitotoxicidade aos 15 DAA, independentemente da taxa de aplicação e da associação do adjuvante (Tabela 1). Em estudo com diferentes cultivares e doses de ioxynil e oxyfluorfen, Oliveira Junior et al. (1995) concluíram que estes herbicidas foram seletivos para a cultura da cebola. A seletividade do herbicida clethodim a cebola já era esperada,

Tabela 1. Fitotoxicidade a cultura da cebola e controle de Cyclospermum leptophyllum e Polygonum persicaria (\%) aos 15 dias após a aplicação. Aurora, SC, 2016.

\begin{tabular}{|c|c|c|c|}
\hline \multicolumn{4}{|c|}{ Fitotoxicidade à cebola (Escala EWRC) } \\
\hline \multirow{2}{*}{ Adjuvante } & \multicolumn{3}{|c|}{ Taxa de aplicação $\left(\mathrm{L} \mathrm{ha}^{-1}\right)$} \\
\hline & 100 & 150 & 200 \\
\hline Sem adjuvante & 1,0 & 1,0 & 1,0 \\
\hline $\mathrm{Nimbus}^{\circledR} 0,5 \% \mathrm{v} \mathrm{v}^{-1}$ & 1,0 & 1,0 & 1,0 \\
\hline \multicolumn{4}{|c|}{ Controle de Cyclospermum leptophyllum (\%) } \\
\hline \multirow{2}{*}{ Adjuvante } & \multicolumn{3}{|c|}{ Taxa de aplicação $\left(\mathrm{L} \mathrm{ha}^{-1}\right)$} \\
\hline & 100 & 150 & 200 \\
\hline Sem adjuvante & $70,2 \quad \mathrm{~A}^{1} \mathrm{a}^{2}$ & $61,6 \mathrm{Aa}$ & $61,2 \mathrm{Ab}$ \\
\hline $\operatorname{Nimbus}^{\circledR} 0,5 \% \mathrm{v} \mathrm{v}^{-1}$ & $73,6 \mathrm{Aa}$ & $72,6 \mathrm{Aa}$ & $77,8 \mathrm{Aa}$ \\
\hline $\mathrm{CV}(\%)$ & & 12,05 & \\
\hline \multicolumn{4}{|c|}{ Controle de Polygonum persicaria (\%) } \\
\hline \multirow{2}{*}{ Adjuvante } & \multicolumn{3}{|c|}{ Taxa de aplicação $\left(\mathrm{L} \mathrm{ha}^{-1}\right)$} \\
\hline & 100 & 150 & 200 \\
\hline Sem adjuvante & $72,4 \quad \mathrm{~A}^{1} \mathrm{a}^{2}$ & $67,2 \mathrm{Aa}$ & $66,0 \mathrm{Aa}$ \\
\hline Nimbus $^{\circledR} 0,5 \% \mathrm{v} \mathrm{v}^{-1}$ & $72,6 \mathrm{Aa}$ & $73,0 \mathrm{Aa}$ & 70,0 Aa \\
\hline CV $(\%)$ & & 12,35 & \\
\hline
\end{tabular}


pois este herbicida é inibidor a enzima ACCase, que sabidamente tem ação específica sobre gramíneas, sendo que sua seletividade a cebola comprovada (Zagonel et al., 2000)

A taxa de aplicação não influenciou no controle de C. leptophyllum (Tabela 1). A adição do adjuvante ao herbicida não teve efeito significativo no controle desta espécie para as taxas de aplicação de 100 e $150 \mathrm{~L} \mathrm{ha}^{-1}$. Todavia, houve melhoria significativa no controle quando se associou o adjuvante a associação de ioxynil + clethodim na taxa de aplicação de $200 \mathrm{~L} \mathrm{ha}^{-1}$. É importante destacar que o controle de C. leptophyllum foi insatisfatório em todos os tratamentos, indicando a limitada eficiência de ioxynil sobre está espécie.

Para a espécie $P$. persicaria, a interação entre taxa de aplicação e adjuvante foi não significativa (Tabela 1). O nível de controle desta planta daninha foi semelhante entre os tratamentos, variando de 66,0 a 73,0\%, indicando desempenho limitado do herbicida ioxynil sobre está espécie.

Os resultados indicaram que a redução na taxa de aplicação, de 200 para $100 \mathrm{~L} \mathrm{ha}^{-1}$, não comprometeu o controle das plantas daninhas avaliadas. Contudo, destaca-se que esses resultados são restritos para estas condições experimentais, já que pesquisas recentes alertaram que a redução na taxa de aplicação, de 187 para $94 \mathrm{~L} \mathrm{ha}^{-1}$, pode comprometer a eficiência dos herbicidas, principalmente quando se reduz a taxa de aplicação sem ajustar os outros parâmetros da aplicação, como o tamanho e espalhamento de gotas e a técnica de aplicação (Meyer et al., 2016).

Não houve diferença significativa para a deposição de calda nas folhas de cebola e em lâminas posicionadas no solo. Contudo, nota-se que em média, houve tendência de maior deposição com a adição de adjuvante (Figuras 2 e 3). A manutenção dos valores de deposição com o aumento da taxa de aplicação pode estar correlacionada com o diâmetro das gotas geradas, já que a elevação da taxa de aplicação e da vazão da ponta aumenta o diâmetro mediano volumétrico (DMV) da população de gotas (Creech et al., 2015). A relação entre
DMV e cobertura é inversa, ou seja, quanto maior o DMV menor será a cobertura proporcionada pela aplicação.

Além disso, esses resultados podem estar associados às características morfológicas da cebola, que se caracteriza pela disposição foliar ereta, folhas com forma cilíndrica, porte baixo e reduzida capacidade de sombreamento do solo (Ferreira et al., 1999; Ferreira et al., 2000; Soares et al., 2003). Essas características

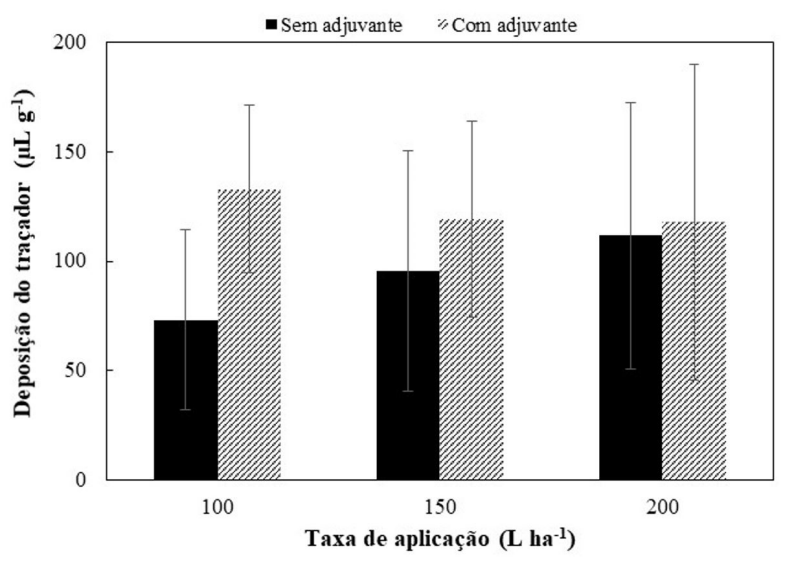

Figura 2. Deposição do traçador azul brilhante (FD\&C 1) em função do uso de adjuvante e da taxa de aplicação na parte aérea da cebola ( $\mu \mathrm{L} \mathrm{g}^{-1}$ de massa seca). Aurora, SC, 2016. Barras indicam o desvio-padrão.

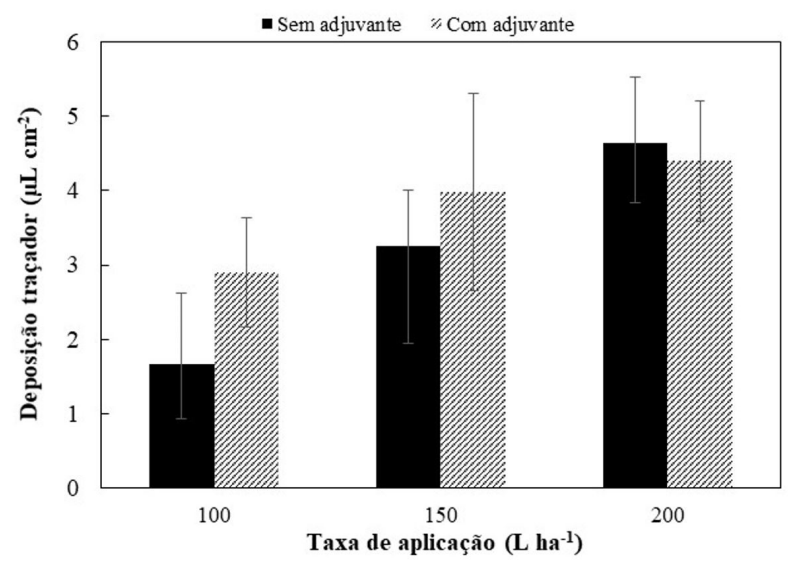

Figura 3. Deposição do traçador azul brilhante (FD\&C 1) em função do uso de adjuvante e da taxa de aplicação em lamínulas ( $\mu \mathrm{L} \mathrm{cm}-2)$. Aurora, SC, 2016. Barras indicam o desvio-padrão. 
contribuem para reduzida taxa de recuperação da calda aplicada e, consequentemente prejudicam a cobertura foliar. Contudo, esperava-se que a deposição da calda nas lamínulas não fosse afetada pela taxa de aplicação e adjuvante, devido as características acima listadas.

Atualmente, a recomendação contida no rótulo do herbicida ioxynil é para utilizar a taxa de aplicação de 200 a 400 L ha $^{-1}$ (Rodrigues e Almeida, 2011). Os resultados desta pesquisa indicam que essa taxa de aplicação pode ser ajustada. A opção por taxa de aplicação excessiva pode ser prejudicial ao meio ambiente, já que além de não contribuir com a eficiência de controle, aumenta os riscos de escorrimento da calda, atingindo locais que não são alvo da aplicação de herbicidas.

Apesar da pouca importância atribuída, o volume de água consumido na aplicação de defensivos é um item importante na composição do custo operacional do tratamento fitossanitário. O fato de o volume de aplicação não ter influência direta no resultado biológico, deve ser considerado já que este contribui com o maior rendimento operacional, redução do número de paradas para reabastecimento, menor consumo de combustível para o transporte de água, maior agilidade na aplicação dos tratamentos fitossanitários e racionalização no uso da água, que é um recurso ambiental que deve ser preservado.

\section{Conclusões}

A taxa de aplicação e a utilização de adjuvante não influenciaram no controle de Cyclospermum leptophyllum e Polygonum persicaria, na fitotoxicidade e na deposição de ioxynil + clethodim. Desta forma, conclui-se que a redução na taxa de aplicação foi uma prática viável e que não comprometeu a eficiência de controle.

\section{Agradecimentos}

Ao Instituto Federal Catarinense Campus Rio do Sul pelo auxílio financeiro.

\section{Referências}

Alves, G.S.; Cunha, J.P.A.R.; Palladini, L.A. Seleção de traçadores para estudo de eficiência das aplicações de produtos fitossanitários. Planta Daninha, v.32, n.4, p.861-870, 2014.

Boller, W.; Machry, M. Efeito da pressão de trabalho e de modelos de pontas de pulverização sobre a eficiência de herbicida de contato em soja. Engenharia Agrícola, v.27, n.3, p.722-727, 2007.

CEMADEN - Centro Nacional de Monitoramento e Alerta de Desastres Naturais. Pluviômetros automáticos. Cachoeira Paulista: CEMADEN, 2018. Disponível em: <https://www.cemaden. gov.br/pluviometros-automatico/>. Acesso em: 27 nov. 2018.

Creech, C.F.; Henry, R.S.; Fritz, B.K.; Kuger, G.R. Influence of herbicide active ingredient, nozzle type, orifice size, spray pressure, and carrier volume rate on spray droplet size characteristics. Weed Technology, v.29, n.2, p.298-310, 2015.

Durigan, J.C.; Silva, M.R.M.; Azania, A.A.P.M. Eficácia e seletividade do herbicida flumioxazin aplicado em pré-emergência na cultura transplantada da cebola. Revista Brasileira de Herbicidas, v.4, n.3, p.11-17, 2005.

EPAGRI. Sistemas de produção para a cebola: Santa Catarina. Florianópolis: Epagri, 2013. 106p.

EWRC - European Weed Research Council. Report of 3rd and 4th meetings of EWRC - Committee of Methods in Weed Research. Weed Research, v.4, p.88, 1964.

Ferreira, L.R.; Durigan, J.C.; Churata-Masca, M.G.C. Eficácia de herbicidas no controle de plantas daninhas na cultura da cebola em semeadura direta. Planta Daninha, v.17, n.1, p.63-72, 1999.

Ferreira, L.R.; Durigan, J.C.; Churata-Masca, M.G.C.; Ferreira, F.A.; Silva, A.A. Seletividade e eficácia da aplicação sequencial de oxyfluorfen e de ioxynil-octanoato, em semeadura direta de cebola. Planta Daninha, v.18, n.1, p.39-50, 2000. 
Gonçalves, P.A.; Palladini, L.A. Eficiência de diferentes bicos e volumes de calda no controle de tripes em cebola. Horticultura Brasileira, v.18, n.2, p.141-144, 2000.

Kuva, M.A.; Salgado, T.P.; Revoredo, T.T.O. Experimentos de eficiência e praticabilidade agronômica com herbicidas. In: Monquero, P.A. Experimentação com herbicidas. São Carlos: Rima, 2016. p. 75-98.

Meyer, C.J.; Norsworthy, J.K.; Kruger, G.R.; Barber, T.L. Effect of nozzle selection and spray volume on droplet size and efficacy of Engenia tank-mix combinations. Weed Technology, v.30, n.2, p.377-390, 2016.

Oliveira, R.B. Caracterização funcional de adjuvantes em soluções aquosas. 2011. 134 f. Tese (Doutorado em Agronomia - Energia na Agricultura) - Universidade Estadual Paulista Júlio de Mesquita, Botucatu, 2011.
Oliveira Junior, R.S.; Silva, J.F.; Ferreira, L.R.; Reis, F.P. Tolerância de três cultivares de cebola ao ioxynil e oxyfluorfen em semeadura direta. Bragantia, v.54, n.2, p.335-342, 1995.

Palladini, L.A.; Raetano, C.G.; Velini, E.D. Choice of tracers for the evaluation of spray deposits. Scientia Agrícola, v.62, n.5, p.440445, 2005.

Rodrigues, B.N.; Almeida, F.S. Guia de herbicidas. 6. ed. Londrina: Edição dos Autores, 2011, 697p. Soares, D.J.; Pitelli, R.A.; Braz, L.T.; Gravena, R.; Toledo, R.E.B. Períodos de interferência das plantas daninhas na cultura de cebola (Allium сера) transplantada. Planta Daninha, v.21, n.3, p.387-396, 2003.

Zagonel, J.; Reghin, M.Y.; Venâncio, W.S. Avaliação de herbicidas de pós-emergência na cultura da cebola. Horticultura Brasileira, v.18, n.3, p.229-231, 2000. 